\title{
A STUDY OF TENSILE STRENGTH OF FRACTURED ROCK MASS BY PHASE FIELD METHOD IN DEAL.II WITH LOCAL REFINEMENT TECHNIQUE
}

\section{Hong Lam Dang}

University of Transport and Communications, No 3 Cau Giay Street, Hanoi, Vietnam

\section{ARTICLE INFO}

TYPE: Research Article

Received: $14 / 8 / 2020$

Revised: $15 / 9 / 2020$

Accepted: 15/9/2020

Published online: 30/9/2020

https://doi.org/10.47869/tcsj.71.7.1

* Corresponding author

Email: dang.hong.lam@utc.edu.vn

\begin{abstract}
Cracking propagation in elastic and porous media is still challenge topics in mechanical, energy, and environmental engineering. In this paper, the phase field method will be used to model the cracking propagation at the small scale for elastic media. This method is doing well in DEAL.II with the help of local refinement technique which allows studying the tensile strength of fractured rock mass behavior without prior knowledge of cracking propagation path and reduction of computational consumption. This implementation is applied to model a fractured rock mass in which a plenty of explicit fractures are distributed though total energy released by Griffith's criterion. Through these applications, we demonstrate and highlight the performance of the phase field method with local refinement technique in modeling crack propagation as well as investigate the tensile strength of fractured rock mass dependency its crack orientation
\end{abstract}

Keywords: fracture propagation, fractured rock mass, explicit modeling of fractures, DEAL.II, phase field method

CC 2020 University of Transport and Communications

\section{INTRODUCTION}

Cracking propagation in elastic and porous media is still challenge topics in mechanical, energy, and environmental engineering. Many approaches for brittle fracture have gained increased interest from studies such as phase-field techniques in [1-5] and extended finite element method (XFEM) in [6]. For assembling cracks / fractures into porous media, 
numerical approach can be applied by incorporated with one of some techniques such as cracks are introduced explicitly, interface elements are used for crack elements or XFEM in which the requirement of cracking trend prior to calculation [6].

Distinguishing to the discontinuity explicit model (like in the XFEM [6]), the lowerdimensional crack surface is approximated by a phase-field function which introduces a diffusive transition zone (brittle or mushy-zone are also common expressions depending on the discipline) between the broken and the unbroken material. In this study, introducing cracks or fractures explicitly is applied. This technique is positive in case grid refinements surrounding cracks do well in DEAL.II [7-10]. For more precise, the theory of cracking propagation in mechanical phenomena was treated largely in the past with a plural of methods. With phase field method based on the Griffith energy [11] and Francfort-Marigo functional [12], Heister et al [1] implemented a treatment of two variables, displacement for linear mechanical behavior and phase field for cracking propagation by fully coupling. The phase field method has an advantage of finding cracking path without understanding of cracking trend during process and can be applied in numerous cracks. However, phase field value of interface domain between cracked area and non-cracked domain is difficult to determine. Base on a series of reports of using phase field method to model cracking propagation supplied by the Institute for Computational Engineering and Sciences (ICES) [15], the paper studies the tensile strength of fractured rock mass in which a plenty of cracks are distributed on the sample.

\section{PHASE FILED METHOD IN DEAL.II}

The mathematical model in cracking propagation involves the coupling of two variables: (a) the linear elasticity system, and (b) the elliptic variational inequality for the phase field variable. Following Griffith's criterion [11] and Francfort-Marigo functional [12] we suppose that crack propagation occurs when the elastic energy restitution rate reaches its critical value $G_{c}$. If $\tau$ is the traction force applied at the part of the boundary $\partial_{N} \Omega$ and it can associate to the crack $\mathrm{C}$, body force $\mathrm{F}$, the following total energy $E(u, C)$ is written as below [1-4]

$$
E(u, C)=\frac{1}{2}(\sigma(u), \mathrm{e}(u))_{\Omega \backslash C}-(\tau, u)_{\partial_{N} \Omega}+(F, \operatorname{div} u)_{\Omega \backslash C}+G_{c} H^{d-1}(\partial C)
$$

We introduce the time-dependent crack phase-field $\varphi$, defined on $\Omega \times(0 ; T)$. The regularized crack functional $\Gamma_{\varepsilon}(\phi)$ reads

$$
\Gamma_{\varepsilon}(\phi)=\frac{1}{2 \varepsilon}\|1-\phi\|^{2}+\frac{\varepsilon}{2}\|\nabla \phi\|^{2}
$$

Note $\varepsilon$ is the aspect ratio of crack $\varepsilon \rightarrow 0$. Thus, we replace the total energy (1) by (2) and neglect the body force $\mathrm{F}=0$, we obtain then

$$
E(u, C)=\frac{1}{2}(\sigma(u), \mathrm{e}(u))_{\Omega \backslash C}-(\tau, u)_{\partial_{N} \Omega}+G_{c}\left(\frac{1}{2 \varepsilon}\|1-\phi\|^{2}+\frac{\varepsilon}{2}\|\nabla \phi\|^{2}\right)
$$

where $G_{c}$ is fracture toughness, $\phi$ is phase field value, $\varepsilon$ is the regularization parameter and it controllers a diffusive transition zone between the broken and unbroken material.

Note that the first part of equation (3), mechanical energy, can be rewritten: 


$$
E(u)=\left(\left((1-\kappa) \phi_{+}^{2}+\kappa\right) \sigma(u), \mathrm{e}(u)\right)
$$

where $\kappa$ is the regularization parameter. It is evident that this parameter should be as small as possible to avoid over-estimation of bulk energy but several of numerical studies observed that $\kappa$ could be set to zero.

The modeling part is based on the fact that the crack can only grow, which is represented by the irreversibility constraint:

$$
\partial_{t}(\phi) \leq 0
$$

\subsection{Local refinement}

In literature, the simple mesh such as the Cartesian mesh will be used in the modeling of fractures. However, in some latest works [1-4], it shown that a quite fine mesh is usually required to satisfy the computational error tolerance and to fit at some acceptable levels the real geometry of fractures. This could lead to very heavy numerical calculation. In order to satisfy the exigencies of the tolerated numerical error, and minimize the number of degree of freedom (D.O.F) of numerical models in many cases local mesh refining strategies (a global refinement would lead to a great number of D.O.F) are proposed in literature. For this aim, a local refinement technique based on the hanging node method is used in this work. It is well known that there are three basic approaches available for the refinement of mesh [12]. The first approach is h-adaptivity in which the mesh connectivity is changed and refined by adding points, thus reducing the size of the cell. The second approach is p-adaptivity which is obtained by increasing or decreasing the order of accuracy of the underlying numerical scheme, thus the mesh connectivity is remained in this second method. The last approach is $r-$ adaptivity in which both the mesh connectivity and order of accuracy are kept constant but the points are repositioned to minimize the computational error. In numerical practice, hadaptivity is the most common used scheme and is known as adaptive mesh refinement.

In DEAL.II the adaptive mesh refinement (called also as local refinement of mesh) is based on the hanging node technique [7-10] [13-14]. This adaptive meshing refinement is the one of the original goals developed in DEAL.II [7-10]. In addition, when performing an adaptive mesh refinement, we usually distinct two kinds of refinement: isotropic and anisotropic refinement [13]. In isotropic mesh refinement, the mesh is refined in all directions (for example one quadrilateral is split into four quadrilaterals) while anisotropic mesh is refined in only one direction (a quadrilateral gets split into two following one direction). Isotropic mesh refinement is provided in DEAL.II. The notion of hanging nodes is used to represent the irregular nodes. If one cell is refined while its neighbor is not refined, the face between the two cells has an irregular point. We recalled the definition of regular and irregular point by [14] in which a point is called regular if it constitutes a vertex (corner) for each of the neighboring cells; otherwise it is irregular. In two-dimensional meshes, the index of irregularity is the maximum number of irregular nodes on an element face: for example, meshes which has the index of irregularity equaled to one are called 1-irregular meshes. In this case, the point connecting the neighbor and the two refined cells is called a "hanging 
node" [13]. An example of a hanging node in 2D is sketched in Fig. 1 in which point 8, 9 are hanging nodes. The 1-irregular meshes were proposed and implemented in DEAL.II.

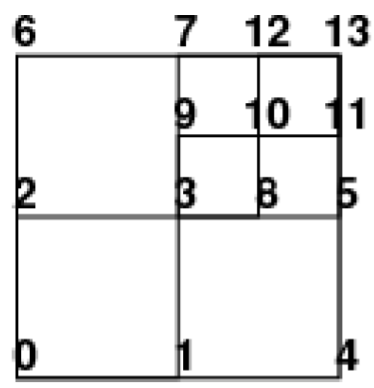

Figure 1. An example of global enumerations of degrees of freedom on the mesh for Q1 elements with each node has one degree of freedom [6].

\subsection{Implementation in DEAL.II}

DEAL.II or Deal.II (Differential Equation Analysis Library) is a free, open library source code to solve the partial differential equations using the finite element method (FEM). Starting from the pioneering work of Numerical Methods Group at Heidelberg University in Germany with the first public release (version 3.0.0) in 2000, this software has received a lot of contributions of the community scientific (http://dealii.org/authors.html) illustrated by hundreds of publications (http://dealii.org/publications.html) in different fields. The current version 9.1.0 is released in 2019. The primary maintainers, coordinating the worldwide development of the library, are today located at Texas A\&M University, Clemson University and Heidelberg University.

DEAL.II is structured in different steps and each step solved a particular problem. As for example, the basic steps 1, 2, 3, 4, 5 and 6 talk about the triangulation, degree of freedom, Laplace matrix, boundary condition, adaptive local refinement and hanging node handing etc which are essential to solve each required problem. Based on these six basic steps, users can implement a simple problem by themselves. From the step 7, DEAL.II classified tutorial into some topics: advance techniques, fluid dynamics, solid mechanics, and time-dependent problems. However, this classification is only reference and users need to understand what they want DEAL.II to supply to solve a problem and what DEAL.II can supply. For a complicated problem, users need to combine two or more steps into a unified code.

Heister et al [1] implemented in this code the phase-field method (which consider two variables: displacement and phase field) to study the cracking-propagation problem in an elastic material by using the fully coupling based on the Griffith energy [11]. The source code is available online at https://github.com/tjhei/cracks. Some other studies of [2-4] about cracking propagation were also conducted in DEAL.II with an extension for the isotropic porous medium. Fig. 2 shows an example of modeling cracks with different level of local refinements by DEAL.II: two times of local refinement with respect to the global mesh (global mesh refinement $=2$ ) is showed on the Fig. 2a and four times of local refinement is presented on the Fig. 2b. The crack size modeled by DEAL.II is reduced from $0.0625 \mathrm{~m}$ to 
$0.015625 \mathrm{~m}$. Hence, the fracture modeled by DEAL.II can be easy to reach the actual crack size by controlling local refinement level.

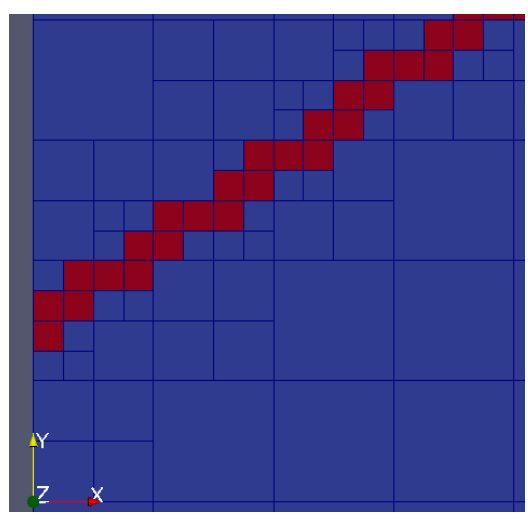

(a)

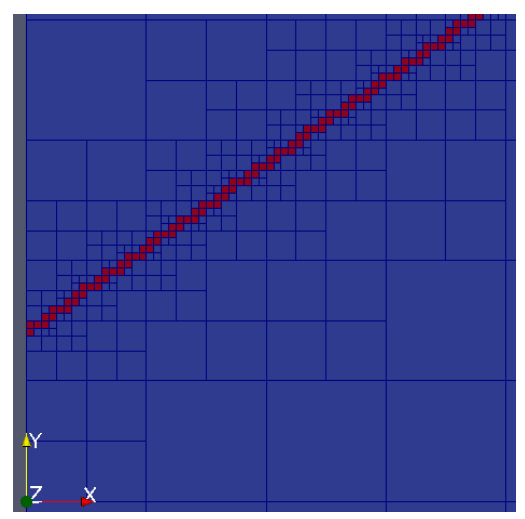

(b)

Figure 2. Fracture elements with the differential level of local refinement: (a) two times of local refinement and (b) four times of local refinement with respect to the global mesh.

\section{INVESTIGATION OF THE TENSILE STRENGTH OF FRACTURED ROCK MASS}

To demonstrate the robustness of the phase field method with local refinement technique, we present two problems of fractured rock mass in pure elasticity in this section. The fractures distributed on this problem come from that the fractures could be uniformed. A plenty of fractures are distributed on this study show the performance of this code. Addition, with this code, the fracture orientation is very easy to carry out by changing the fracture angle on the code.

\subsection{Multi fractures under tension load}

We consider a $1 \mathrm{~m}$-square problem where a constant tension displacement $u_{y}$ is applied on the top (boundary No.2) while a fixed boundary is applied on the bottom (boundary No.1) (Fig. 3a). The Elastic modulus $E$ and Poisson ratio $v$ of intact rock are $10^{4} \mathrm{MPa}$ and 0.2 , respectively. The fracture toughness $G_{c}$ is assumed as $10^{-4} \mathrm{Mpa} \cdot \sqrt{\mathrm{m}}$. In this problem, the crack intensity $\left(\mathrm{p}_{21}\right)$ is $5 \mathrm{~m} / \mathrm{m}^{2}$ where the crack intensity is defined by equation $p_{21}=\frac{\sum l_{i}}{S}$ in which $l_{i}$ is length of cracks $i$ and $S$ is total area of sample. The sketch of crack distribution is presented on the Fig. $3 b$ where the cracks are oriented by $\theta$ degree. Following steps is used to generate samples

Step 1: Generate location of crack center from the number of cracks $N_{p}=100$ and crack intensity $\left(\mathrm{p}_{21}=5 \mathrm{~m} / \mathrm{m}^{2}\right)$ and the inclined angle $(\theta=0)$ for sample of $1 \mathrm{~m}$ square (Fig. $\left.4 \mathrm{a}\right)$. Note that all cracks are not intersected themselves.

Step 2: Length of cracks, $l$, will be uniformed and calculated by $l=p_{21} S / N_{p}$ (Fig. 4b) 
Step 3: Other samples will be created by rotating all cracks at sample center with $\theta$ degree (Fig. 4c)

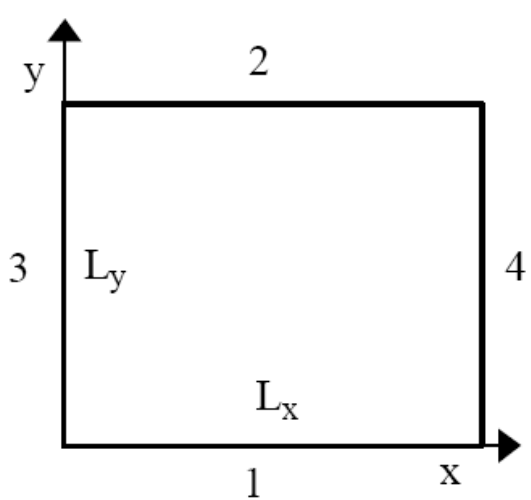

(a)

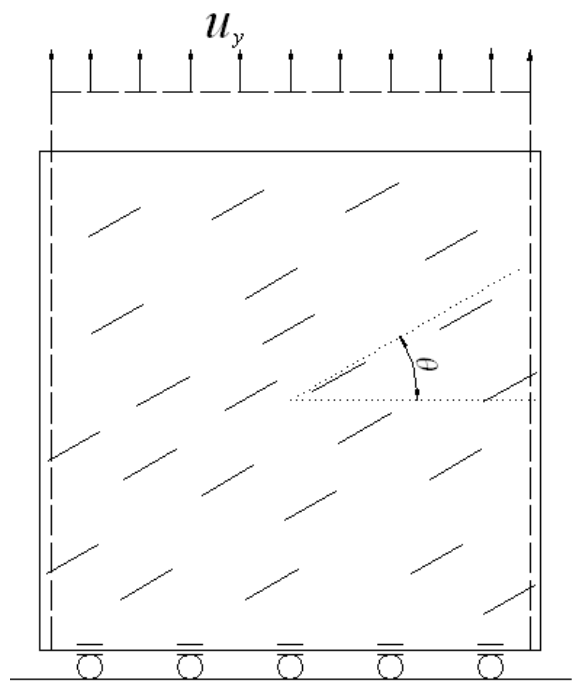

(b)

Figure 3. (a) Boundary identification and (b) Sketch of crack distribution.

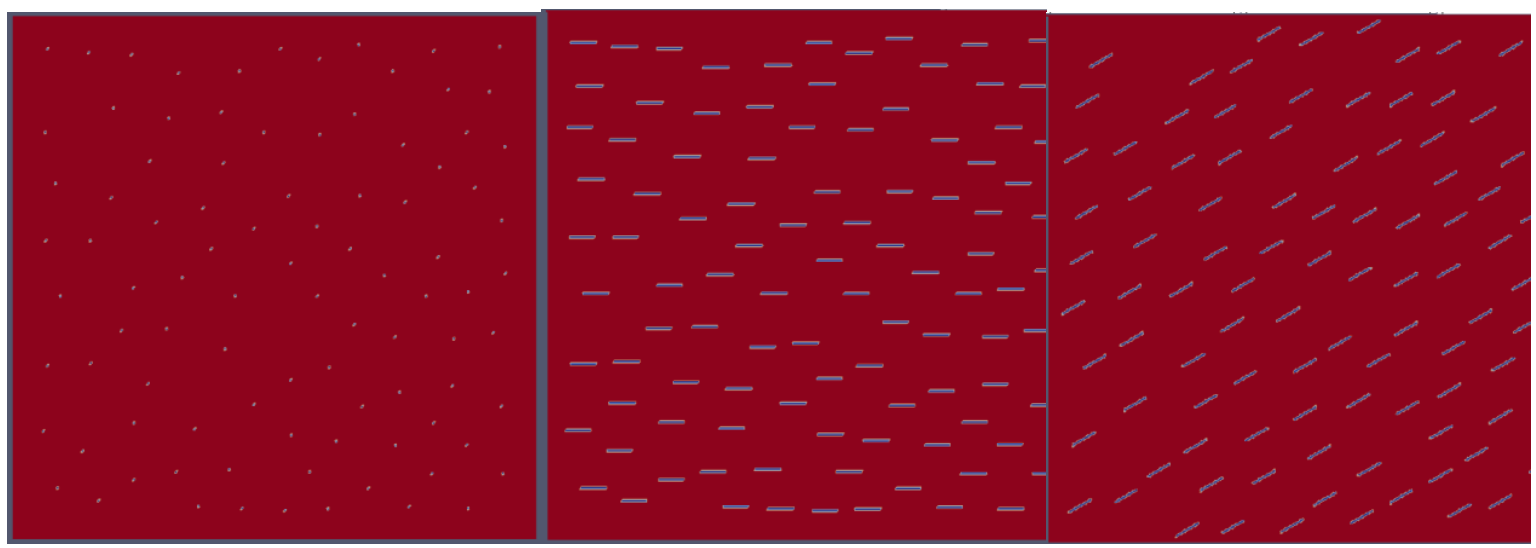

(a)

(b)

(c)

Figure 4. Steps to generate samples: (a) crack center generation; (b) sample with 0 degree crack angle; (c) sample with different crack angle.

As mentioned in [1], the mesh size, $h$, needs to be satisfied the condition $h \leq \varepsilon$. In this calculation, we choice $\varepsilon=4 h$ and $\kappa=h^{*} 10^{-10}$. In these tests, we obtain the maximum tension $u_{y}$ on surface No.2. Here, the parameters of phase field are: the regularization parameters are: $\varepsilon=0.011$ and mesh size $h \leq \varepsilon$, we choose $h=\frac{\varepsilon}{4}=0.00276$ and $\kappa=h^{*} 10^{-10}=2.76 * 10^{-10}$. Thus, number of refined mesh in following calculations is $n=9$

Fig. 5 is the propagation of sample under increasing tension vertical displacement. Three interval steps are figured out in Fig. 5. Fig. 5a is initial step when cracks are not propagating and blue elements are cracks while red ones are non cracks. The cracks are starting to propagate in the Fig. $5 \mathrm{~b}$ and those cracks are continuing to the Fig. 5c. 

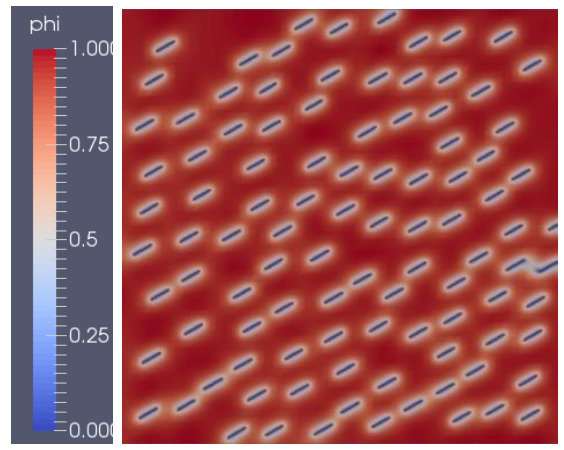

(a)

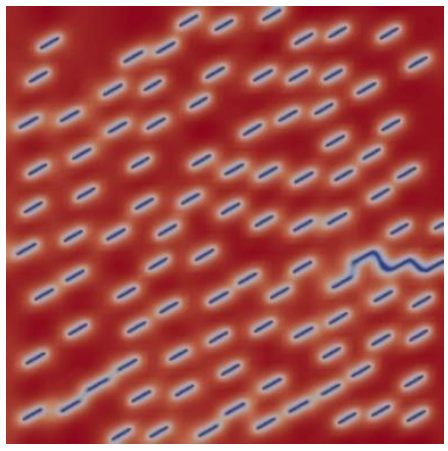

(b)

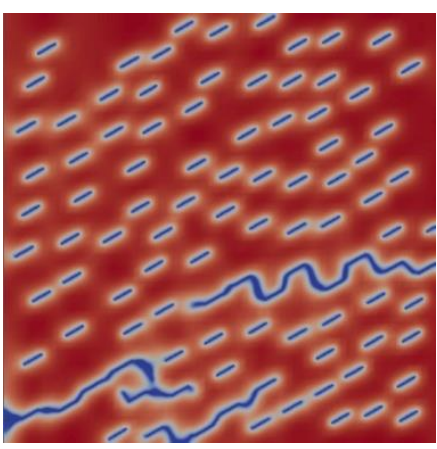

(c)

Figure 5. Crack propagation at interval steps: (a) initial step; (b) crack propagation; (c) final step.

\subsection{Tensile strength of fractured rock mass depended on fracture orientation}

The second result in this paper is on studying the effect of fracture orientation to the tensile strength of fractured rock mass. Seven kinds of crack orientation are studied from "0 degree" for horizontal cracks to "90 degree" for vertical cracks with 15 degree interval. With the purpose of investigating the fracture effect to the tensile strength of fractured rock mass, the ratio of tensile strength of fracture rock mass compared with the tensile strength of intact rock is expressed. The results of seven above cases are sketched in the Fig. 6a and Fig. 6b corresponding to tensile test in two directions: vertical direction and horizontal direction, respectively. The tensile strength, the peak value of each tensile test, occurred at around $0.025 \mathrm{~mm}$ displacement. We can see that the tensile strength of fractured rock mass is depended on the fracture orientation (Fig. 7). For tensile test in vertical direction, the tensile strength is about $20 \%$ of the tensile strength of intact rock for horizontal fracture case while it is increase to $60 \%$ of the tensile strength of intact rock for vertical fracture case. In contrast, tensile test in horizontal direction, the tensile strength is approximated to the tensile strength of intact rock for horizontal fracture case and it is decrease to $20 \%$ of the tensile strength of intact rock for vertical fracture case

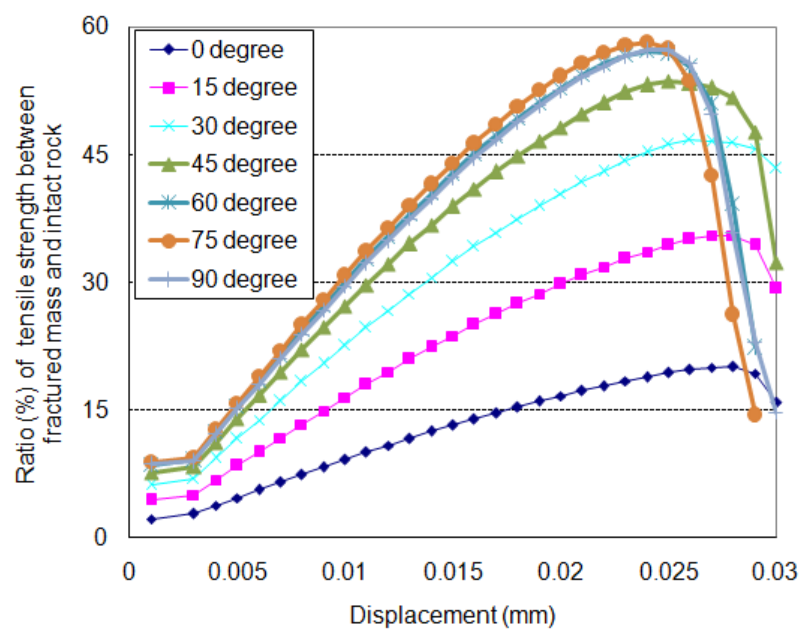

(a)

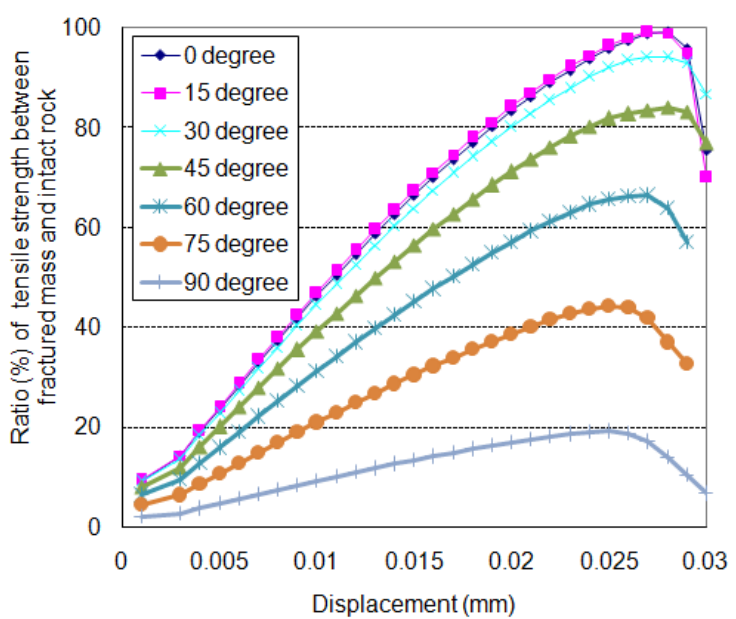

(b)

Figure 6. Ratio of tensile strength between fractured rock mass and intact rock during tensile test in (a) vertical direction (b) horizontal direction. 


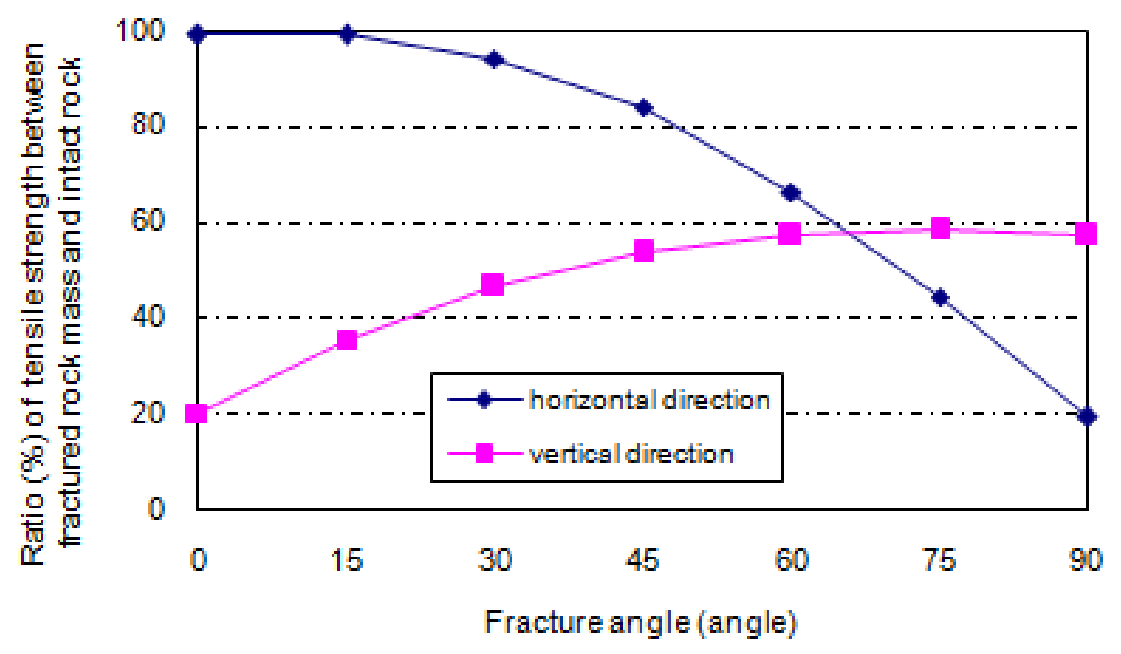

Figure 7. Percentage of tensile strength of fractured rock mass compared its intact rock.

\section{CONCLUSION}

An example of cracking propagation in the mechanical brittle behavior for multi fractures was studied by phase field method with the help of local refinement in open source code DEAL.II. This method allows to take into account a plenty of fractures into one model as well as to get the cracking path of these fractures under tension load. Based on this study, the effect properties of fractured rock mass could be evaluated incorporated with cracking propagation (stress-dependency permeability of fractured rock mass). Though a case study, we figured out that tensile strength of fractured rock mass depended significantly on fracture orientation.

\section{ACKNOWLEDGEMENTS}

The author would like to thank to Prof. Dashnor HOXHA and Dr. Duc Phi DO of University of Orleans (France) and my colleagues of the University of Transport and Communications (Vietnam) for all supports on this work.

\section{REFERENCES}

[1] T. Heister, M.F. Wheeler, T. Wick, A primal-dual active set method and predictor-corrector mesh adaptivity for computing fracture propagation using a phase-field approach, Comput. Methods Appl. Mech. Engrg., 290 (2015) 466-495. https://doi.org/10.1016/j.cma.2015.03.009

[2] A. Mikelic, M.F. Wheeler, T. Wick, Phase-field modeling of a fluid-driven fracture in a poroelastic medium, Computational Geosciences, 19 (2015) 1171-1195. https://doi.org/10.1007/s10596-015-9532-5

[3] A. Mikelic, M.F. Wheeler, T. Wick, A phase-field method for propagation fluid-filled fractures coupled to a surrounding porous medium, Multiscale Modeling and Simulation, 13 (2015) 367-398. https://doi.org/10.1137/140967118

[4] T. Wick, G. Singh, M.F. Wheeler, Fluid-filled fracture propagation with a phase-field approach and coupling to a reservoir simulator, SPE Journal, 21 (2015) 19 pages. https://doi.org/10.2118/168597-PA

[5] P. M. Pham, Anynasys free vibration of the functionally grade material cracked plates with varying thickness using the Phase-field theory, Transport and Communications Science Journal, 70 (2019) 122-131. (in Vietnamese) https://doi.org/10.25073/tcsj.70.2.35 
[6] T. Mohammadnejad, A.R. Khoei, Hydro-mechanical modeling of cohesive crack propagation in multiphase porous media using the extended finite element method, Int. J. Numer. Anal. Meth. Geomech., 37 (2013) 1247-1279. https://doi.org/10.1002/nag.2079

[7] W. Bangerth, G. Kanschat, Concepts for object-oriented finite element software - the Deal.II library, Preprint 1999-43, SFB 359, Heidelberg (1999).

[8] W. Bangerth, R. Hartmann, G. Kanschat, Deal.II- a general purpose object oriented finite element library, ACM Trans. Math. Software, 33 (2007). https://doi.org/10.1145/1268776.1268779

[9] H.L. Dang, A hydro-mechanical modeling of double porosity and double permeability fractured reservoirs, PhD thesis, University of Orleans, France, 2018.

[10] H.L., Dang, P.H, Thinh, A methodology of re-generating a representative element volume of fractured rock mass, Transport and Communications Science Journal, 71 (2020) 347-358. https://doi.org/10.25073/tcsj.71.4.4

[11] A.A. Griffith, The phenomena of rupture and flow in solids, Philos. Trans. R. Soc. Lond., 221 (1921) 163-198. http://mbarkey.eng.ua.edu/courses/AEM644/Griffith1921fracture.pdf

[12] G. Francfort, J.-J. Marigo, Revisiting brittle fracture as an energy minimization problem, J. Mech. Phys. Solids, 46 (1998) 1319-1342. https://doi.org/10.1016/S0022-5096(98)00034-9

[13] J. Karlsson, Implementing Anisotropic Adaptive Mesh Refinement in OpenFOAM, Master's Thesis in Computer Science, Algorithms, Languages and Logic, Chalmers University of Technology, Göteborg, Sweden (2012).

[14] L. Demkowicz, J. Oden, W. Rachowicz, O. Hardy, Toward a universal h-p adaptive finite element strategy, part 1. Constrained approximation and data structure, Computer Methods in Applied Mechanics and Engineering, 77 (1989) 79-112. https://doi.org/10.1016/0045-7825(89)90129-1 